Research Article

\title{
Stunting and Associated Factors among Under-Five-Age Children in West Guji Zone, Oromia, Ethiopia
}

\author{
Eyob Afework, ${ }^{1}$ Selamawit Mengesha, ${ }^{1}$ and Demelash Wachamo $\mathbb{D}^{2}$ \\ ${ }^{1}$ Department of Public Health, College of Medicine and Health Sciences, Hawassa University, Hawassa, Ethiopia \\ ${ }^{2}$ Department of Public Health, Hawassa College of Health Sciences, Sidama National Regional State, Hawassa, Ethiopia \\ Correspondence should be addressed to Demelash Wachamo; demmenew1@gmail.com
}

Received 12 September 2020; Revised 4 December 2020; Accepted 18 January 2021; Published 4 February 2021

Academic Editor: Abdah Md Akim

Copyright (C) 2021 Eyob Afework et al. This is an open access article distributed under the Creative Commons Attribution License, which permits unrestricted use, distribution, and reproduction in any medium, provided the original work is properly cited.

Background. Stunting is one of the most important public health problems in Ethiopia. It remains a problem of greater magnitude particularly in rural and low-income areas. It reflects chronic nutritional deficiencies and illness that occur during the most critical periods for growth and development in early life. It needs proper intervention to save the future, unless it resulted in diminished cognitive and physical development for the rest of their lives. Therefore, this study aimed to assess the prevalence of stunting and associated factors among under-five children in West Guji Zone, Oromia, Ethiopia. Method. A community-based cross-sectional study was conducted among 767 under-five children who were included in this study by using a multistage sampling technique in 12 kebeles from 3 selected districts. Data were collected from a mother/caregiver of the child by using a structured pretested questionnaire. Standardized anthropometric measurements were used to measure length, weight, and height of a child. Data were entered into Epi Info software version 3.5.1 and exported to SPSS version 23 for analysis for descriptive and logistic regression models. Result. The prevalence of stunting was 244 (31.8\%) with 95\% CI (28.6-35.2) among under-five-age children. The underfive children whose fathers had a polygamous marriage ( $\mathrm{AOR}=4.92,95 \% \mathrm{CI}$ : 3.46, 7.00), being female sex $(\mathrm{AOR}=1.74,95 \% \mathrm{CI}$ : $1.23,2.47)$, having below 4 meal frequencies $(\mathrm{AOR}=2.95,95 \% \mathrm{CI}: 1.56,5.58)$, not vaccinated (AOR $=1.75,95 \% \mathrm{CI}: 1.15,2.67)$, and from poor households' wealth status $(\mathrm{AOR}=3.03,95 \% \mathrm{CI}: 1.63,5.63)$ and also from severely food insecure household $(\mathrm{AOR}=2.92,95 \% \mathrm{CI}: 1.36,6.24)$ were short for their age compared with their counterparts. Conclusion. Nearly one-third of the under-five children were stunted in the study area which needs intervention on child-feeding practice to avoid sex discrimination in the community. In addition to this health officials in collaboration with other sectors, it is needed to act together to improve enforcement of the law for polygamous marriage, the household's wealth status, and food security for the better health of a child and future.

\section{Background}

Stunting is when a child has a low height for their age, usually due to malnutrition, repeated infections, and poor social stimulation [1]. It indicates a community reveals chronic undernutrition and inadequate nutrients for a prolonged period that begins in the womb [2, 3]. Stunting (chronic undernutrition) is one of the major public health concerns that a significant number of children are suffering from moderate-to-severe forms, and the problem is more prominent in countries that are underdeveloped [4].

The 2018 Global Nutrition Report shows there are 150.8 million (22.2\%) children under five years are stunted [5]. In
Africa, there are 58.7 million under-five children who are stunted [5]. It accounts for 39 percent of stunted under-five children globally. Five regions of Africa have a stunting prevalence of more than $30 \%$ including Eastern Africa which has $36.7 \%$ of stunting prevalence [6]. Sub-Saharan Africa remains the region with the highest under-five mortality rate in the world in 2017, 76 deaths/ 1,000 live births [7], and 34\% prevalence of stunting [8].

However, Ethiopia working towards the "Seqota" Declaration aims to end hunger and undernutrition and to attain the Sustainable Development Goals (SDGs) target to cut stunting by $40 \%$ by 2030 [9] and the WHO in stunting target by 2025 [10]. The prevalence of stunting has decreased 
considerably, from $51 \%$ in 2005 to $37 \%$, wasting decreased from $12 \%$ to $7 \%$, and underweight children were from $33 \%$ to $21 \%$ over these 14 years in 2019 [11]. Stunting is one of the most important public health problems in Ethiopia with a double burden of undernutrition comprising both wasted and stunted under-five children. Most studies indicated that stunting is associated with low socioeconomic status, low educational level of parents, poor water supply and sanitation, and high infectious disease burden [12-14].

The basic and major food source for the study area was "Enset," and there was high population density and polygamous marriage was common in the study area. Furthermore, the study area mainly produces cash crop farming such as coffee and khat which were not used for household consumption. It is also important to target specific intervention on the production and utilization of diversified food. In addition to this, it needs to understand major influencing factors to target and intervein the impact of the stunting. Particularly, in the study area, there was the gap of the study to target the interventions based on the contributing factors such as low educated mothers, polygamous marriage, high family size, low income, food insecurity, male sex child preference, infectious disease, diarrheal disease, and other environmental related factors. Hence, it is very important to see the prevalence of stunting and its determinant factors among under-five-age children to reduce the impact of malnutrition. These study findings can be helpful evidence in planning sound interventions to reduce malnutrition among infant and young children and its determinant factors which can be used for public health officials, clinicians, and health planners to reduce the impacts of malnutrition.

\section{Methods}

2.1. Study Setting and Design. This study was conducted in the West Guji Zone, Oromia Region, located in the southern part of Ethiopia. It is located $365 \mathrm{~km}$ from the capital Addis Ababa in the main road of Addis Ababa. The zonal administration was composed of a total population of $1,241,797$ according to the West Guji Zone health and demographic surveillance department dataset. There are also 10 districts and 144 kebeles in the West Guji Zone. Among this, the number of children under 5 was estimated at 193,845 . The main crops produced in the area are cash crop such as coffee, maize, and teff which is produced in some parts of the district. Enset (false banana) is the staple food, and many households consume it. The study design was a community-based cross-sectional study that was conducted from June 01, 2019, to January 2020.

2.2. Study Population and Eligibility Criteria. All 6- to 59month-aged children were the source of population, while all randomly selected children aged 6-59 months and available on the study period were considered as the study population. Those mothers who were unable to respond due to their seriously ill child were excluded from the study.

\subsection{Study Variables}

Outcome Variable. Stunting was determined by computing the height-for-age $\mathrm{z}$-score (HAZ) of less than -2 standard deviations (SDs).

Exposure Variables. They were sociodemographic and economic variables, obstetric and other maternal characteristics, child morbidity and vaccination status, child-caring practice, and environmental health characteristics.

2.4. Sample Size and Sampling Procedures. The sample size (n) was calculated using the following single-population proportion formula based on the assumption of $(p) 37 \%$ of children under 5 are short for their age or stunted (below -2 SD) from the 2019 Ethiopia Mini Demographic and Health Survey (EMDHS) [11], 95\% confidence interval (CI) (1.96), $5 \%$ margin of error (d), design effect (2), and adding $10 \%$ contingency:

$$
\begin{aligned}
n & =\left(Z_{(1-\alpha / 2)}\right)^{2} \frac{P[1-P]}{d^{2}}=\frac{(1.96)^{2}(0.52)(0.48)}{(0.05)^{2}} \\
& =(358.2 \times 2)=716.4+10 \%(71.64)=788.02 \approx 788 .
\end{aligned}
$$

Therefore, the required sample size was $n=788$ included in the study.

The selection of the study participants, the sampling frame prepared from the West Guji Zone health and demographic surveillance department dataset, which contains child date of birth (age), kebele, household identification, household head name, marital status, and child name, was used. The sample was proportionally allocated to randomly selected 12 kebeles out of 36 kebeles from 3 selected districts.

2.5. Data Collection Tools and Procedures. The data were collected through an interview by pretested and structured questionnaires. Sociodemographic and other determinants were assessed. The questionnaires were translated into the local language and validated before the study time was done outside of the study area, and necessary modifications were done based on the findings. Anthropometric measurements such as HFA z-score/LFA z-score were collected from the selected children using a height measuring board to assess stunting. A survey was carried out by trained data collectors and nurses. Principal investigators and supervisors follow the data collection process and check them for consistency and completeness. Mothers of under-five-year aged children were asked to list out food groups and drinks consumed by their children in the previous $24 \mathrm{hrs}$ before the survey. Mothers of children were asked to count their children's meal frequency in the past $24 \mathrm{hrs}$. [15]. In order to recall the foods and drinks consumed, and meal frequency in the past $24 \mathrm{hrs}$, the questionnaires were pretested and validated on $5 \%$ of the $6-59$-month children before two weeks in the study time outside the study area. Then, some modifications to the sequence and arrangement of multiple answer questionnaires were made. Data collectors were five clinical 
nurses supervised by one Bachelor of Science (B.Sc.) nurse supervisor and investigators. Training and practical demonstrations on interview techniques and physical measurement procedures were given to data collectors for two consecutive days assessed for competency.

2.6. Data Analysis. Data entry, cleaning, and analysis were done by SPSS V. 23. Descriptive analysis including frequency distribution and the percentage was made to determine the prevalence of the stunting, to describe socioeconomic and demographic and other determinants. Bivariate logistic regression analysis was conducted for crude odds ratio (COR), and all factors with a $p$ value $<0.25$ were the candidate to a multivariable logistic regression to control confounding effects. The Hosmer-Lemeshow goodness-offit statistic was used to assess whether the necessary assumptions for the application of multiple logistic regression are fulfilled. Adjusted odds ratios (AORs) with 95\% confidence intervals (CIs) were used to measure the strength of the association between outcome variables and its determinant factors. Finally, $p$ value $<0.05$ declared a significant association.

\subsection{Operational Definitions}

2.7.1. Stunting. Children are short for an age means that children have an HAZ of less than -2 SD on the WHO growth standard chart [16].

2.7.2. Minimum Meal Frequency. Minimum is defined as the proportion of children aged 6-59 months, who receive solid, semisolid, or soft foods at the minimum numbers of two and three times for children aged 6-8 months and 9-59 months, respectively [15].

2.7.3. Minimum Dietary Diversity Score. It is the proportion of infants and young children aged 6-59 months who received foods and drinks from 4 and more food groups in the previous $24 \mathrm{hrs}$. Consumption of any amount and quality of food from each food group was sufficient to "count" [15-17].

2.7.4. Kebele. It has the smallest administrative structure with a household number of nearly 500 .

\section{Results}

3.1. Sociodemographic Characteristics. A total of 767 participants were interviewed, yielding a response rate of $97.3 \%$. The mean of the participant's mother in the completed year's age was 29 with a standard deviation of $( \pm 6.34)$ years, and 405 (52.8\%) of them were aged between 25 and 34 years old. Majority, 721 (94.0\%), of the mothers were married, and 511 (66.6\%) were housewives. Regarding the educational status of the mother, 399 (52.0\%) had an elementary school, while 178 (23.2\%) had no formal education. Majority, 581 (75.7\%), of the under-five-year aged children's family had more than 5 family members. In addition to this, more than one-third,
277 (36.1\%), of them were from children whose fathers had a polygamous marriage. Three hundred five (42.4\%) of underfive-year aged children's families were poor in wealth status. Regarding the household food security, 584 (76.1\%) were mild/moderately insecure and $65(8.5 \%)$ were severely insecure. Out of the under-five-year aged children participated, nearly half $392(51.1 \%)$ were aged between 25 and 47 months and 389 (50.7\%) were male (Table 1$)$.

3.2. Healthcare and Other Related Characteristics. Out of 767 participants, $462(60.2 \%)$ and 247 (32.2\%) used piped water as a source of drinking water outside the compound or communal and inside their compound, respectively, while $34(4.4 \%)$ and $20(2.6 \%)$ still used protected spring and surface water, respectively. The majority of $728(94.9 \%)$ had functional toilet facilities. Regarding the healthcare utilization-related factors, 144 (18.8\%) of mothers had no antenatal care (ANC) follow-up and only $53(6.9 \%)$ attended ANC4. In addition to this, 287 (37.4\%) still had the experience of home delivery. Regarding the under-five-year aged children, the majority, 583 (76.0\%), were vaccinated, and among these, $468(80.3 \%)$ of them were fully vaccinated. Nearly one-third of 255 (33.2\%) had diarrhea in the last two weeks and 138 (54.1\%) sought modern healthcare. Most, 755 (98.4\%), of them had breastfeeding, and 751 (97.9\%) start breastfeeding with in the 1st one hour. The majority of 618 (80.6\%) were breastfed for more than 18 months, while nearly half $368(48.0 \%)$ of them started at 6 months of complementary feeding and 409 (54.9\%) were fed by their hand (Table S1).

3.3. Dietary Diversity Score of Mothers. The mean dietary diversity score of food groups was 5.24 with a standard deviation $( \pm \mathrm{SD})$ of \pm 1.41 . The under-five-year aged children consumed the following: $736(96.0 \%)$ consumed grains, white roots, and tubers, $442(57.6 \%)$ consumed pulses such as beans, peas, and lentils, $614(80.1 \%)$ consumed milk and milk product, and 738 (96.2\%) consumed dark green leafy vegetables (Table S2).

3.4. The Prevalence of Stunting. The prevalence of stunting was $244(31.8 \%)$ with 95\% CI (28.6-35.2) among under-fiveage children scored a $z$-score below $-2 \mathrm{SD}$ of the WHO standards or short for their age. Out of the study participants, 91 (11.9\%) were severely stunted and 153 (19.9\%) were moderately stunted (Figure 1). The prevalence of the stunting was varied, $101(26.0 \%)$ vs. $143(37.8 \%)$ among males and females, respectively.

3.5. Factors Affecting Stunting. The under-five children whose mother had no formal education and above 5 family members had a significant association with the bivariable logistic regression analysis, while others remain statistically significant after controlling for confounder at multivariable logistic regression. The under-five children whose fathers had a polygamous marriage were 3 times $(\mathrm{AOR}=4.92,95 \%$ CI: $3.46,7.00$ ) short for their age or stunted than them whose 
TABle 1: Sociodemographic characteristics of respondents, West Guji Zone, Oromia, Ethiopia, 2020.

\begin{tabular}{|c|c|}
\hline Category & No. $(\%)$ \\
\hline \multicolumn{2}{|l|}{ Age of the mother } \\
\hline $18-24$ & $191(24.9)$ \\
\hline $25-34$ & $405(52.8)$ \\
\hline 35 or above & $171(22.3)$ \\
\hline \multicolumn{2}{|l|}{ Marital status } \\
\hline Unmarried & $46(6.0)$ \\
\hline Married & $721(94.0)$ \\
\hline \multicolumn{2}{|l|}{ Occupation of mother } \\
\hline Housewife & $511(66.6)$ \\
\hline Merchant & $167(21.8)$ \\
\hline Employed and others* & $89(11.6)$ \\
\hline \multicolumn{2}{|l|}{ Educational status of father } \\
\hline No formal education & $178(23.2)$ \\
\hline Primary education & $399(52.0)$ \\
\hline Secondary and above & $190(24.8)$ \\
\hline \multicolumn{2}{|l|}{ Maternal education } \\
\hline No formal education & $280(36.5)$ \\
\hline Primary education & $370(48.2)$ \\
\hline Secondary and above & $117(15.3)$ \\
\hline \multicolumn{2}{|l|}{ Family size } \\
\hline$\leq 5$ & $186(24.3)$ \\
\hline$>5$ & $581(75.7)$ \\
\hline \multicolumn{2}{|l|}{ Husband had polygamy } \\
\hline No & $490(63.9)$ \\
\hline Yes & $277(36.1)$ \\
\hline \multicolumn{2}{|l|}{ Household's wealth } \\
\hline Wealthy & $139(18.1)$ \\
\hline Medium & $303(39.5)$ \\
\hline Poor & $325(42.4)$ \\
\hline \multicolumn{2}{|l|}{ Household food insecurity } \\
\hline Food secure & $118(15.4)$ \\
\hline Mild/moderately insecure & $584(76.1)$ \\
\hline Severely insecure & $65(8.5)$ \\
\hline \multicolumn{2}{|l|}{ Age of child } \\
\hline$\leq 24$ & $244(31.8)$ \\
\hline $25-47$ & $392(51.1)$ \\
\hline $48-59$ & $131(17.1)$ \\
\hline \multicolumn{2}{|l|}{ Sex of child } \\
\hline Male & $389(50.7)$ \\
\hline Female & $378(49.3)$ \\
\hline
\end{tabular}

$*$ Daily laborer and students.

fathers had a monogamous marriage. Furthermore, a child being female sex (AOR $=1.74,95 \%$ CI: 1.23, 2.47) was shorter than males for their age. A child who was not vaccinated $(\mathrm{AOR}=1.75,95 \% \mathrm{CI}: 1.15,2.67)$ was shorter than vaccinated children. A child who had less than four meal frequencies ( $\mathrm{AOR}=2.95,95 \% \mathrm{CI}$ : 1.56, 5.58), a child from poor households' wealth status ( $\mathrm{AOR}=3.03,95 \% \mathrm{CI}$ : 1.63 , 5.63) and medium households' wealth status $(\mathrm{AOR}=2.07$, $95 \%$ CI: 1.10, 3.87), and a child from severely food insecure household $(\mathrm{AOR}=2.92,95 \% \mathrm{CI}$ : $1.36,6.24)$ were short for their age or stunted compared with their counterparts (Table 2).

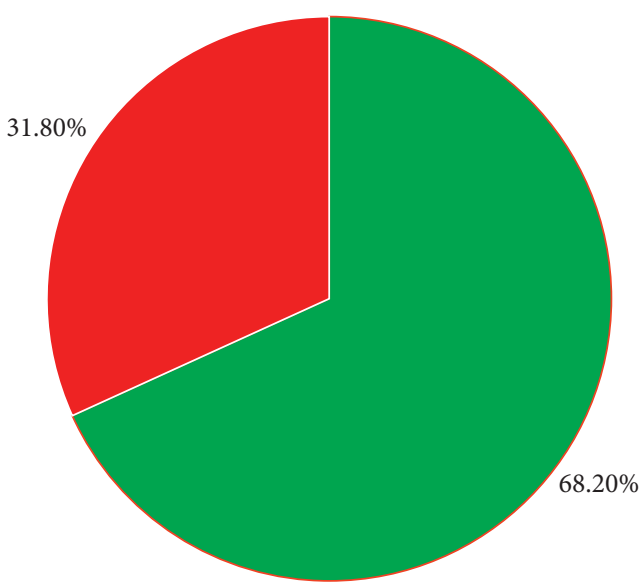

Normal

Stunted

FIGURE 1: The prevalence of stunting among under-five children in the West Guji Zone, Oromia, Ethiopia, 2020.

\section{Discussion}

This community-based cross-sectional study revealed that the prevalence of stunting among under-five-age children was (31.8\%) with 95\% CI (28.6-35.2), and they scored a z-score below $-2 \mathrm{SD}$ or are short for their age. Out of the study participants, $11.9 \%$ were severely stunted and $19.9 \%$ were moderately stunted which is lower than the national prevalence of $37 \%$ of children under 5 who are short for their age or stunted (below -2 SD) from the 2019 EMDHS [11], 39.3\% in Boricha Woreda/District, Southern Ethiopia [18], 47.9\% in Arba Minch, Southern Ethiopia [13], 43.9\% in Democratic Republic of Congo [19], 37\% in Nepal [20], and $43.2 \%$ in Bangladesh [21]. This difference might be sociodemographic factors, low awareness, and other seasonal and cultural differences in ways of traditional food preparations among the study population [22]. This indicates that there are some improvements in the prevalence of stunting among underfive-age children which gives hope to attain the WHO and SDGs targets. This result higher than the previous study reported as follows: Hawassa Zuria District, Southern Ethiopia (26.6\%) [23], Jima Zone, Southwest Ethiopia (24.1\%) [24], Sodo Zuria District in South Ethiopia (24.9\%) [12], the urban-rural gradient in Eastern Ethiopia (26.9\%) [25], Adwa Town of North Ethiopia (12.2\%) [26], and Brazil (29.9\%) [20]. This can explain the variations between the different parts of the country, and this may be due to the different types of the interventions particularly by nongovernmental organizations and the agricultural activities, child-feeding practice, and the difference in socioeconomic activities. This may be due to a lack of nearby market access and lack of media access to get different advice about dietary diversity.

The study finding revealed that mothers who were not working currently, had any formal education, had more than 5 family size, and cannot feed their children minimum meal frequency had shorter children than their counterparts. This 
TAвLE 2: Bivariable and multivariable logistic regression analyses for stunting among under-five-age children in West Guji Zone, Oromia, Ethiopia, 2020.

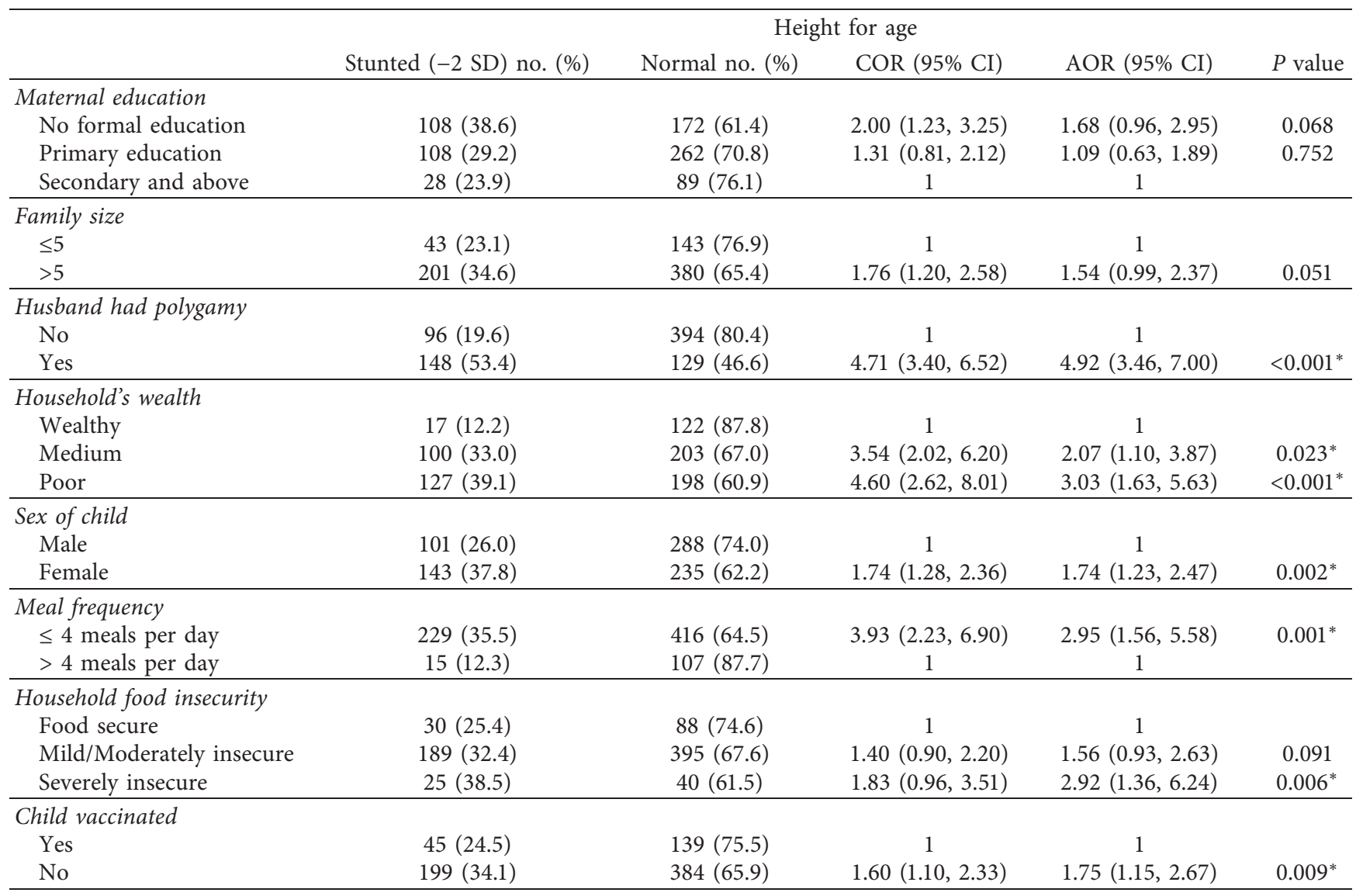

can be explained that the under-five children were most vulnerable to stunting due to low or poor family wealth status, low educational level, frequent round delivery or high family size, food insecurity of the household, and male sex preference of the household which were major determinants of the stunting among under-five children at the study area.

The study results revealed that under-five children whose fathers had a polygamous marriage were shorter for their age or stunted than them whose fathers had a monogamous marriage. This study result agreed with Kenya [27]. The polygamous marriage is commonly practiced in the study area and may favor a child to undernutrition and stunting among under-five children. In the case of the study area, most of the women are economically dependent on their husbands and they cannot afford the cost of well-nourished, minimum meal frequency, and dietary diversity on their own unless they are supported by their husbands. This implies that government bodies need to improve enforcement of the law for polygamy. It also needs better strategies to strengthen women's education and create job opportunities for women to reduce stunting among under-five children.

Furthermore, a child being female sex was shorter than males for their age, similarly reported from Rwanda and Sodo Zuria District, South Ethiopia [12]. This may be due to cultural issues, gender preference, and discrimination during the feed of their children. Despite having the above result, the study from Tigray Region, Northern Ethiopia, reported being a male sex child who was shorter than females for their age [28]. This difference may be due to the difference in socioeconomic and cultural acceptance of the gender in the community and maternal education, and the urban population may also have better exposure to mass media and have better information about gender equality.

A child who had less than four meal frequencies was more likely stunted than those who had more than four meal frequencies which agrees with Sodo Zuria District, South Ethiopia [12], and Northeast Ethiopia [29]. This may be due to that low food and nutrition intake leads to undernutrition and stunting. Health officials and clinicians need to emphasize pertinent health education on gender preference and discrimination during the feed of their children.

A child who was not vaccinated was shorter for their age or stunted compared with vaccinated children. This study result agreed with the previous study report from India [30]. It is also associated with infectious disease and diarrheal morbidity [31]. This might be preventive effect of the vaccine especially infectious disease and diarrhea which leads to malnutrition. Mothers who had not vaccinated their children also may be related with poor child care and feeding practice due to less access to mass media and to appropriate messages and campaigns on heath and vaccines. This needs 
better strategies to address mothers who had low income and had no formal education who live in shanty houses in the city.

A child from poor wealth status and severely food insecure households was shorter for their age or stunted compared with their counterparts, similarly reported from Arba Minch, Southern Ethiopia [13], Dabat, Ethiopia, and Boricha District, Southern Ethiopia [18]. This can be explained that children who were born in poor wealth status and severely food insecure household were facing a challenge to get food due to the number of the priorities to feed all under-five children and competition with elder children, and in low-income households, that may lead to low access to adequate dietary intake in kinds and the amounts. This implies that government bodies and other stakeholders should work together to improve the household wealth status and create job opportunities for women and maintain affirmative actions for women employment. It also needs better strategies to strengthen women's education and employment in the study area.

4.1. Limitations. There might be a potential for recall and social desirability bias on the child-feeding practice and socioeconomic. In addition to this, since the study is crosssectional, it may not be strong to demonstrate a direct cause and effect relationship between risk factors and outcome. Since the study included children aged 24-59 months, there is a potential recall bias among respondents answering questions relating to events happening in the past two years and above, taking an extra meal during pregnancy or lactation and dietary diversity of children in the past $24 \mathrm{hrs}$.

\section{Conclusions}

This study result shows that nearly one-third of the underfive children are still short for their age or stunted. The under-five children whose fathers had a polygamous marriage, being a female sex child, had less than 4 meal frequencies/day, and being poor in wealth status and severely food insecure household were short for their age or stunted compared with their counterparts. It needs intervention on factors which attributes to stunting by providing health education on family planning and healthy child-feeding practices to combat or minimize gender-based discrimination among children at the community. In addition to this health officials in collaboration with other sectors, it is needed to act together to improve enforcement of the law for polygamous, the household's wealth status, and food security for the better health of a child and future. It also needs better strategies to strengthen women's education and employment to reduce stunting among under-five children.

\section{Abbreviations}

ANC: Antenatal care

AOR: Adjusted odds ratio

COR: Crude odds ratio

DDS: Dietary diversity score

EMDHS: Ethiopia Mini Demographic and Health Survey
ETB: $\quad$ Ethiopian birr

IYCF: Infant and young child feeding

SDGs: $\quad$ Sustainable Development Goals

SPSS: $\quad$ Statistical Package for Social Science

UNICEF: United Nations International Children's Emergency Fund

WHO: World Health Organization.

\section{Data Availability}

The data used to support the findings are included within the article and available from the corresponding author upon request.

\section{Ethical Approval}

Ethical clearance was obtained from Hawassa University College of Medicine and Health Sciences, conducted following the Declaration of Helsinki, and approved by the institutional review board or ethics committee. A support letter was obtained from the West Guji Zone health department to each selected district.

\section{Consent}

All participants were informed well about the purpose and the procedures of the study. All responses were kept confidential and anonymous. Participation was fully voluntary, and written informed consent was obtained from each participant.

\section{Disclosure}

The funder had no role in designing the study and conducting the analysis.

\section{Conflicts of Interest}

The authors declare no conflicts of interest.

\section{Authors' Contributions}

EA, SM, and DW wrote the conception and were responsible for study design, execution, acquisition of data, analysis, and interpretation, and they read and approved the final manuscript. All the authors contributed to data analysis, drafting or revising the article, and gave final approval of the version to be published.

\section{Acknowledgments}

The study was funded by Hawassa University.

\section{Supplementary Materials}

Supplementary Table S1: healthcare and environmental characteristics of respondents and Table S2: dietary diversity score of respondents, West Guji Zone, Oromia, Ethiopia, 2020. Supplementary File 2: English questionnaires and assessment tools used to assess prevalence of stunting and 
associated factors among under-five-age children. (Supplementary Materials)

\section{References}

[1] C. P. Stewart, L. Iannotti, K. G. Dewey, K. F. Michaelsen, and A. W. Onyango, "Contextualising complementary feeding in a broader framework for stunting prevention," Maternal and Child Nutrition, vol. 9, pp. 27-45, 2013.

[2] A. J. Prendergast and J. H. Humphrey, "The stunting syndrome in developing countries," Paediatrics and International Child Health, vol. 34, no. 4, pp. 250-265, 2014.

[3] R. Julie, W. Keri, and W. Rebecca, Cases in Global Health Delivery Malnutrition Concept Note, Harvard Business Publishing, Boston, MA, USA, 2015.

[4] L. M. G. Cruz, G. G. Azpeitia, D. R. Súarez et al., "Factors associated with stunting among children aged 0 to 59 Months from the central region of Mozambique," Nutrients, vol. 9, no. 5, 451 pages, 2017.

[5] Development Initiatives Poverty Research Ltd, Global Nutrition Report Shining a Light to Spur Action on Nutrition, Development Initiatives Poverty Research Ltd, Bristol, UK, 2018.

[6] World Bank, Stunting Reduction in Sub-saharan Africa, World Bank, 2018.

[7] United Nations Children's Fund, World Health Organization and the World Bank Group.Global and Regional Aggregates Are for the Year, Levels and Trends in Child malnutrition. UNICEF/WHO/World Bank Group Joint Child Malnutrition Estimates, United Nations Children's Fund, World Health Organization and the World Bank Group.Global and Regional Aggregates Are for the Year, New York, Geneva and Washington (DC), 2018.

[8] UNICEF, The State of the World's Children, UNICEF, New York, NY, USA, 2017.

[9] Federal Democratic Republic of Ethiopia, National Nutrition Program 2016-2020, Federal Democratic Republic of Ethiopia, Addis Ababa, Ethiopia, 2016.

[10] UNICEF, Reducing Stunting in Children under 5 Years of Age: A Comprehensive Evaluation of Unicef's Strategies and Programme Performance Global Synthesis Report, UNICEF, New York, NY, USA, 2017.

[11] Ethiopian Public Health Institute (EPHI) [Ethiopia] and ICF, Ethiopia Mini Demographic and Health Survey 2019: Key Indicators, EPHI and ICF, Rockville, MA, USA, 2019.

[12] S. K. Dake, F. B. Solomon, T. M. Bobe, H. A. Tekle, and E. G. Tufa, "Predictors of stunting among children 6-59 months of age in Sodo Zuria District, South Ethiopia: a community based cross-sectional study," BMC Nutrition, vol. 5, p. 23, 2019.

[13] B. Bogale, B. T. Gutema, and Y. Chisha, "Prevalence of stunting and its associated factors among children of 6-59 Months in Arba minch health and demographic surveillance site (HDSS), southern Ethiopia: a community-based crosssectional study," Journal of Environmental Public Health, vol. 2020, Article ID 9520973, 8 pages, 2020.

[14] M. Kahssay, E. Woldu, A. Gebre, and S. Reddy, "Determinants of stunting among children aged 6 to 59 months in pastoral community, Afar region, North East Ethiopia: unmatched case control study," BMC Nutrition, vol. 6, p. 9, 2020.

[15] World Health Organization, Indicators for Assessing Infant and Young Child Feeding Practices, WHO Press, Geneva, Switzerland, 2010.
[16] World Health Organization, Child Growth Standards: Growth Velocity Based on Weight, Length and Head Circumference: Methods and Development, World Health Organization, Geneva, Switzerland, 2009.

[17] World Health Organization, Indicators for Assessing Infant and Young Child Feeding Practices, WHO Press, Geneva, Switzerland, 2008.

[18] A. Yoseph and H. Beyene, "The high prevalence of intestinal parasitic infections is associated with stunting among children aged 6-59 months in Boricha Woreda, Southern Ethiopia: a cross-sectional study," BMC Public Health, vol. 20, no. 1, p. 1270, 2020.

[19] N.-B. Kandala, T. P. Madungu, J. B. Emina, K. P. Nzita, and F. P. Cappuccio, "Malnutrition among children under the age of five in the Democratic Republic of Congo (DRC): does geographic location matter?" BMC Public Health, vol. 11, no. 1, p. 261, 2011.

[20] A. A. Ferreira, J. R. Welch, R. V. Santos, S. A. Gugelmin, and C. E. Coimbra, "Nutritional status and growth of indigenous Xavante children, Central Brazil," Nutrition Journal, vol. 11, no. 1, p. 3, 2012.

[21] M. Mridha, C. Chaparro, S. Matias et al., Acceptability of Lipid-Based Nutrient Supplements and Micronutrient Powders Among Pregnant and Lactating Women and Infants and Young Children in Bangladesh and Their Perceptions about Malnutrition and Nutrient Supplements, p. 360, FHI, Washington, DC, 2012.

[22] M. Belayneh, E. Loha, and B. Lindtjørn, "Seasonal variation of household food insecurity and household dietary diversity on wasting and stunting among young children in A drought prone area in South Ethiopia: a cohort study," Ecology of Food and Nutrition, pp. 1-26, 2020.

[23] B. B. Desalegn, E. Kinfe, K. Fikre, and T. Bosha, "Stunting and its associated factors in under five years old children: the case of Hawassa University Technology villages, Southern Ethiopia," Journal of Environmental Science, Toxicology and Food Technology, vol. 10, no. 11, pp. 25-31, 2016.

[24] K. H. Abate and T. Belachew, "Care and not wealth is a predictor of wasting and stunting of 'The Coffee Kids' of Jimma Zone, southwest Ethiopia," Nutrition and Health, vol. 23, no. 3, pp. 193-202, 2017.

[25] A. A. Irenso, Y. Dessie, Y. Berhane, N. Assefa, C. R. Canavan, and W. W. Fawzi, "Prevalence and predictors of adolescent linear growth and stunting across the urban-rural gradient in eastern Ethiopia," Tropical Medicine \& International Health, vol. 25, no. 1, pp. 101-110, 2020.

[26] T. Gebregyorgis, T. Tadesse, and A. Atenafu, "Prevalence of thinness and stunting and associated factors among adolescent school girls in Adwa town, North Ethiopia," International Journal of Food Science, vol. 2016, p. 8323982, 2016.

[27] J. Okanda, G. Otieno, J. Kinuthia, P. Kohler, and G. JohnStewart, "Higher likelihood of 6-months exclusive breastfeeding among HIV infected than uninfected mothers: a household survey in Kenya," International Breastfeeding Journal, vol. 13, p. 51, 2018.

[28] B. T. Woldeamanuel and T. T. Tesfaye, "Risk factors associated with under-five stunting, wasting, and underweight based on Ethiopian demographic health survey datasets in Tigray region, Ethiopia," Journal of Nutrition and Metabolism, vol. 2019, Article ID 6967170, 11 pages, 2019.

[29] B. Getnet, M. Solomon, and S. Mekonnen, "Prevalence of stunting and associated actors among preschool children: a ommunity based comparative cross ectional study in Ethiopia," BMC Nutrition, vol. 4, no. 28, 2018. 
[30] I. Meshram, K. Mallikharjun Rao, N. Balakrishna et al., "Infant and young child feeding practices, sociodemographic factors and their association with nutritional status of children aged $<3$ years in India: findings of the National Nutrition Monitoring Bureau survey," 2011-2012. Public Health Nutrition, vol. 22, no. 1, pp. 104-114, 2019.

[31] G. Shumetie, M. Gedefaw, A. Kebede, and T. Derso, "Exclusive breastfeeding and rotavirus vaccination are associated with decreased diarrheal morbidity among under-five children in Bahir Dar, northwest Ethiopia," Public Health Reviews, vol. 39, p. 28, 2018. 